\title{
When the Riemann Hypothesis might be false
}

\author{
Frank Vega
}

the date of receipt and acceptance should be inserted later

\begin{abstract}
Robin criterion states that the Riemann Hypothesis is true if and only if the inequality $\sigma(n)<e^{\gamma} \times n \times \log \log n$ holds for all natural numbers $n>5040$, where $\sigma(n)$ is the sum-of-divisors function and $\gamma \approx 0.57721$ is the Euler-Mascheroni constant. Let $q_{1}=2, q_{2}=3, \ldots, q_{m}$ denote the first $m$ consecutive primes, then an integer of the form $\prod_{i=1}^{m} q_{i}^{a_{i}}$ with $a_{1} \geq a_{2} \geq \cdots \geq a_{m} \geq 0$ is called an Hardy-Ramanujan integer. If the Riemann Hypothesis is false, then there are infinitely many HardyRamanujan integers $n>5040$ such that Robin inequality does not hold and $n<$ $(4.48311)^{m} \times N_{m}$, where $N_{m}=\prod_{i=1}^{m} q_{i}$ is the primorial number of order $m$.
\end{abstract}

Keywords Riemann hypothesis · Robin inequality · sum-of-divisors function · prime numbers

Mathematics Subject Classification (2010) MSC 11M26 · MSC 11A41 · MSC $11 \mathrm{~A} 25$

\section{Introduction}

In mathematics, the Riemann Hypothesis is a conjecture that the Riemann zeta function has its zeros only at the negative even integers and complex numbers with real part $\frac{1}{2}$ [4]. As usual $\sigma(n)$ is the sum-of-divisors function of $n$ [2]:

$$
\sum_{d \mid n} d
$$

where $d \mid n$ means the integer $d$ divides to $n$ and $d \nmid n$ means the integer $d$ does not divide to $n$. Define $f(n)$ to be $\frac{\sigma(n)}{n}$. Say Robins $(n)$ holds provided

$$
f(n)<e^{\gamma} \times \log \log n .
$$

F. Vega

CopSonic, 1471 Route de Saint-Nauphary 82000 Montauban, France

ORCiD: 0000-0001-8210-4126

E-mail: vega.frank@gmail.com 
The constant $\gamma \approx 0.57721$ is the Euler-Mascheroni constant, and $\log$ is the natural logarithm. The importance of this property is:

Theorem 1.1 If the Riemann Hypothesis is false, then there are infinitely many natural numbers $n>5040$ such that Robins $(n)$ does not hold [4].

We recall that an integer $n$ is said to be square free if for every prime divisor $q$ of $n$ we have $q^{2} \nmid n$ [2]. Robins $(n)$ holds for all natural numbers $n>5040$ that are square free [2]. In addition, we show that Robins $(n)$ holds for some $n>5040$ when $\frac{\pi^{2}}{6} \times$ $\log \log n^{\prime} \leq \log \log n$ such that $n^{\prime}$ is the square free kernel of the natural number $n$. Let $q_{1}=2, q_{2}=3, \ldots, q_{m}$ denote the first $m$ consecutive primes, then an integer of the form $\prod_{i=1}^{m} q_{i}^{a_{i}}$ with $a_{1} \geq a_{2} \geq \cdots \geq a_{m} \geq 0$ is called an Hardy-Ramanujan integer [2]. Based on the theorem 1.1, we know this result:

Theorem 1.2 If the Riemann Hypothesis is false, then there are infinitely many natural numbers $n>5040$ which are an Hardy-Ramanujan integer and Robins $(n)$ does not hold [2].

We prove if the Riemann Hypothesis is false, then there are infinitely many HardyRamanujan integers $n>5040$ such that Robins $(n)$ does not hold and $n<(4.48311)^{m} \times$ $N_{m}$, where $N_{m}=\prod_{i=1}^{m} q_{i}$ is the primorial number of order $m$.

\section{A Central Lemma}

These are known results:

Lemma 2.1 [2]. For $n>1$ :

$$
f(n)<\prod_{q \mid n} \frac{q}{q-1} .
$$

Lemma $2.2[3]$.

$$
\prod_{k=1}^{\infty} \frac{1}{1-\frac{1}{q_{k}^{2}}}=\zeta(2)=\frac{\pi^{2}}{6}
$$

The following is a key lemma. It gives an upper bound on $f(n)$ that holds for all natural numbers $n$. The bound is too weak to prove $\operatorname{Robins}(n)$ directly, but is critical because it holds for all natural numbers $n$. Further the bound only uses the primes that divide $n$ and not how many times they divide $n$.

Lemma 2.3 Let $n>1$ and let all its prime divisors be $q_{1}<\cdots<q_{m}$. Then,

$$
f(n)<\frac{\pi^{2}}{6} \times \prod_{i=1}^{m} \frac{q_{i}+1}{q_{i}} .
$$

Proof We use that lemma 2.1:

$$
f(n)<\prod_{i=1}^{m} \frac{q_{i}}{q_{i}-1}
$$


Now for $q>1$,

$$
\frac{1}{1-\frac{1}{q^{2}}}=\frac{q^{2}}{q^{2}-1} .
$$

So

$$
\begin{aligned}
\frac{1}{1-\frac{1}{q^{2}}} \times \frac{q+1}{q} & =\frac{q^{2}}{q^{2}-1} \times \frac{q+1}{q} \\
& =\frac{q}{q-1} .
\end{aligned}
$$

Then by lemma 2.2,

$$
\prod_{i=1}^{m} \frac{1}{1-\frac{1}{q_{i}^{2}}}<\zeta(2)=\frac{\pi^{2}}{6} .
$$

Putting this together yields the proof:

$$
\begin{aligned}
f(n) & <\prod_{i=1}^{m} \frac{q_{i}}{q_{i}-1} \\
& \leq \prod_{i=1}^{m} \frac{1}{1-\frac{1}{q_{i}^{2}}} \times \frac{q_{i}+1}{q_{i}} \\
& <\frac{\pi^{2}}{6} \times \prod_{i=1}^{m} \frac{q_{i}+1}{q_{i}} .
\end{aligned}
$$

\section{A Particular Case}

We can easily prove that $\operatorname{Robins}(n)$ is true for certain kind of numbers:

Lemma 3.1 Robins $(n)$ holds for $n>5040$ when $q \leq 5$, where $q$ is the largest prime divisor of $n$.

Proof Let $n>5040$ and let all its prime divisors be $q_{1}<\cdots<q_{m} \leq 5$, then we need to prove

$$
f(n)<e^{\gamma} \times \log \log n
$$

that is true when

$$
\prod_{i=1}^{m} \frac{q_{i}}{q_{i}-1} \leq e^{\gamma} \times \log \log n
$$

according to the lemma 2.1. For $q_{1}<\cdots<q_{m} \leq 5$,

$$
\prod_{i=1}^{m} \frac{q_{i}}{q_{i}-1} \leq \frac{2 \times 3 \times 5}{1 \times 2 \times 4}=3.75<e^{\gamma} \times \log \log (5040) \approx 3.81 .
$$

However, we know for $n>5040$

$$
e^{\gamma} \times \log \log (5040)<e^{\gamma} \times \log \log n
$$

and therefore, the proof is complete when $q_{1}<\cdots<q_{m} \leq 5$. 
4 Helpful Lemmas

For every prime number $p_{n}>2$, we define the sequence $Y_{n}=\frac{e^{\frac{1}{2 \times \log \left(p_{n}\right)}}}{\left(1-\frac{1}{\log \left(p_{n}\right)}\right)}$.

Lemma 4.1 For every prime number $p_{n}>2$, the sequence $Y_{n}$ is strictly decreasing.

Proof For every real value $x \geq 3$, we state the function

$$
f(x)=\frac{e^{\frac{1}{2 \times \log (x)}}}{\left(1-\frac{1}{\log (x)}\right)}
$$

which is equivalent to

$$
f(x)=g(x) \times h(u)
$$

where $g(x)=e^{\frac{1}{2 \times \log (x)}}$ and $h(u)=\frac{u}{u-1}$ for $u=\log (x)$. We know that $g(x)$ decreases as $x \geq 3$ increases, Moreover, we note that $h(u)$ decreases as $u>1$ increases where $u=$ $\log (x)>1$ for $x \geq 3$. In conclusion, we can see that the function $f(x)$ is monotonically decreasing for every real value $x \geq 3$ and therefore, the sequence $Y_{n}$ is monotonically decreasing as well. In addition, $Y_{n}$ is essentially a strictly decreasing sequence, since there is not any natural number $n>1$ such that $Y_{n}=Y_{n+1}$.

In mathematics, the Chebyshev function $\theta(x)$ is given by

$$
\theta(x)=\sum_{p \leq x} \log p
$$

where $p \leq x$ means all the prime numbers $p$ that are less than or equal to $x$.

Lemma 4.2 [5]. For $x \geq 41$ :

$$
\theta(x)>\left(1-\frac{1}{\log (x)}\right) \times x .
$$

Besides, we know that

Lemma 4.3 [5]. For $x \geq 286$ :

$$
\prod_{q \leq x} \frac{q}{q-1}<e^{\gamma} \times\left(\log x+\frac{1}{2 \times \log (x)}\right) .
$$

We will prove another important inequality:

Lemma 4.4 Let $q_{1}, q_{2}, \ldots, q_{m}$ denote the first $m$ consecutive primes such that $q_{1}<$ $q_{2}<\cdots<q_{m}$ and $q_{m}>286$. Then

$$
\prod_{i=1}^{m} \frac{q_{i}}{q_{i}-1}<e^{\gamma} \times \log \left(Y_{m} \times \theta\left(q_{m}\right)\right)
$$


Proof From the theorem 4.2, we know that

$$
\theta\left(q_{m}\right)>\left(1-\frac{1}{\log \left(q_{m}\right)}\right) \times q_{m} .
$$

In this way, we can show that

$$
\begin{aligned}
\log \left(Y_{m} \times \theta\left(q_{m}\right)\right) & >\log \left(Y_{m} \times\left(1-\frac{1}{\log \left(q_{m}\right)}\right) \times q_{m}\right) \\
& =\log q_{m}+\log \left(Y_{m} \times\left(1-\frac{1}{\log \left(q_{m}\right)}\right)\right) .
\end{aligned}
$$

We know that

$$
\begin{aligned}
\log \left(Y_{m} \times\left(1-\frac{1}{\log \left(q_{m}\right)}\right)\right) & =\log \left(\frac{e^{\frac{1}{2 \times \log \left(q_{m}\right)}}}{\left(1-\frac{1}{\log \left(q_{m}\right)}\right)} \times\left(1-\frac{1}{\log \left(q_{m}\right)}\right)\right) \\
& =\log \left(e^{\frac{1}{2 \times \log \left(q_{m}\right)}}\right) \\
& =\frac{1}{2 \times \log \left(q_{m}\right)} .
\end{aligned}
$$

Consequently, we obtain that

$$
\log q_{m}+\log \left(Y_{m} \times\left(1-\frac{1}{\log \left(q_{m}\right)}\right)\right) \geq\left(\log q_{m}+\frac{1}{2 \times \log \left(q_{m}\right)}\right) .
$$

Due to the theorem 4.3, we prove that

$$
\prod_{i=1}^{m} \frac{q_{i}}{q_{i}-1}<e^{\gamma} \times\left(\log q_{m}+\frac{1}{2 \times \log \left(q_{m}\right)}\right)<e^{\gamma} \times \log \left(Y_{m} \times \theta\left(q_{m}\right)\right)
$$

when $q_{m}>286$.

\section{Proof of Main Theorems}

The next theorem implies that Robins $(n)$ holds for a wide range of natural numbers $n>5040$.

Theorem 5.1 Let $\frac{\pi^{2}}{6} \times \log \log n^{\prime} \leq \log \log n$ for some $n>5040$ such that $n^{\prime}$ is the square free kernel of the natural number $n$. Then Robins $(n)$ holds.

Proof Let $n^{\prime}$ be the square free kernel of the natural number $n$. Let $n^{\prime}$ be the product of the distinct primes $q_{1}, \ldots, q_{m}$. By assumption we have that

$$
\frac{\pi^{2}}{6} \times \log \log n^{\prime} \leq \log \log n
$$


For all square free $n^{\prime} \leq 5040$, Robins $\left(n^{\prime}\right)$ holds if and only if $n^{\prime} \notin\{2,3,5,6,10,30\}$ [2]. However, Robins $(n)$ holds for all natural numbers $n>5040$ when $n^{\prime} \in\{2,3,5,6,10,15,30\}$ due to the lemma 3.1. When $n^{\prime}>5040$, we know that Robins $\left(n^{\prime}\right)$ holds and so

$$
f\left(n^{\prime}\right)<e^{\gamma} \times \log \log n^{\prime} .
$$

By the previous lemma 2.3:

$$
f(n)<\frac{\pi^{2}}{6} \times \prod_{i=1}^{m} \frac{q_{i}+1}{q_{i}} .
$$

Suppose by way of contradiction that $\operatorname{Robins}(n)$ fails. Then

$$
f(n) \geq e^{\gamma} \times \log \log n .
$$

We claim that

$$
\frac{\pi^{2}}{6} \times \prod_{i=1}^{m} \frac{q_{i}+1}{q_{i}}>e^{\gamma} \times \log \log n .
$$

Since otherwise we would have a contradiction. This shows that

$$
\frac{\pi^{2}}{6} \times \prod_{i=1}^{m} \frac{q_{i}+1}{q_{i}}>\frac{\pi^{2}}{6} \times e^{\gamma} \times \log \log n^{\prime} .
$$

Thus

$$
\prod_{i=1}^{m} \frac{q_{i}+1}{q_{i}}>e^{\gamma} \times \log \log n^{\prime}
$$

and

$$
\prod_{i=1}^{m} \frac{q_{i}+1}{q_{i}}>f\left(n^{\prime}\right)
$$

This is a contradiction since $f\left(n^{\prime}\right)$ is equal to

$$
\frac{\left(q_{1}+1\right) \times \cdots \times\left(q_{m}+1\right)}{q_{1} \times \cdots \times q_{m}} .
$$

Theorem 5.2 If the Riemann Hypothesis is false, then there are infinitely many HardyRamanujan integers $n>5040$ such that Robins $(n)$ does not hold and $n<(4.48311)^{m} \times$ $N_{m}$, where $N_{m}=\prod_{i=1}^{m} q_{i}$ is the primorial number of order $m$.

Proof Let $\prod_{i=1}^{m} q_{i}^{a_{i}}$ be the representation of some natural number $n>5040$ as a product of primes $q_{1}<\cdots<q_{m}$ with natural numbers as exponents $a_{1}, \ldots, a_{m}$. The primes $q_{1}<\cdots<q_{m}$ must be the first $m$ consecutive primes and $a_{1} \geq a_{2} \geq \cdots \geq a_{m} \geq 0$ since the natural number $n>5040$ could be an Hardy-Ramanujan integer. We assume that Robins $(n)$ does not hold. Indeed, we know there are infinitely many HardyRamanujan integers such as $n>5040$ when the Riemann Hypothesis is false according to the theorem 1.2. From the lemma 4.4, we know that

$$
\prod_{i=1}^{m} \frac{q_{i}}{q_{i}-1}<e^{\gamma} \times \log \left(Y_{m} \times \theta\left(q_{m}\right)\right)=e^{\gamma} \times \log \log \left(N_{m}^{Y_{m}}\right)
$$


when $q_{m}>286$. In this way, if Robins $(n)$ does not hold, then $n<N_{m}^{Y_{m}}$ since by the lemma 2.1 we have that

$$
f(n)<\prod_{i=1}^{m} \frac{q_{i}}{q_{i}-1} .
$$

That is the same as $n<N_{m}^{Y_{m}-1} \times N_{m}$. We can check that $q_{m}^{Y_{m}-1}$ is monotonically decreasing for all primes $q_{m}>286$ due to the lemma 4.1. Certainly, the function

$$
g(x)=x^{\left(\frac{e^{\frac{1}{2 \times \log (x)}}}{\left(1-\frac{1}{\log (x)}\right)}-1\right)}
$$

complies that its derivative is lesser than zero for all real numbers $x>286$. Indeed, a function $g(x)$ of a real variable $x$ is monotonically decreasing in some interval if the derivative of $g(x)$ is lesser than zero and the function $g(x)$ is continuous over that interval [1]. We know that $q_{m}$ could comply with $q_{m} \geq 1000000$ ! for infinitely many Hardy-Ramanujan integers $n>5040$ such that Robins $(n)$ does not hold, where $(\ldots)$ ! is the factorial function. Certainly, if $q_{m}$ would have an upper bound by some positive value, then there would not be infinitely many natural numbers $n>5040$ which are an Hardy-Ramanujan integer and Robins $(n)$ does not hold because of the theorem 5.1. Consequently, it is enough to show that

$$
q_{m}^{Y_{m}-1} \leq g(1000000 !)<4.48311
$$

for all primes $q_{m} \geq 1000000$ !. Moreover, we would obtain that

$$
q_{m}^{Y_{m}-1}>q_{j}^{Y_{m}-1}
$$

for every integer $1 \leq j<m$. Finally, we can state that $n<(4.48311)^{m} \times N_{m}$ since $N_{m}^{Y_{m}-1}<(4.48311)^{m}$ when $n>5040$ could be any of the infinitely many HardyRamanujan integers such that Robins $(n)$ does not hold and $q_{m} \geq 1000000$ !.

\section{Acknowledgments}

I thank Richard J. Lipton and Craig Helfgott for helpful comments.

\section{References}

1. Anderson, G., Vamanamurthy, M., Vuorinen, M.: Monotonicity Rules in Calculus. The American Mathematical Monthly 113(9), 805-816 (2006). DOI 10.1080/00029890.2006.11920367

2. Choie, Y., Lichiardopol, N., Moree, P., Solé, P.: On Robin's criterion for the Riemann hypothesis. Journal de Théorie des Nombres de Bordeaux 19(2), 357-372 (2007). DOI doi:10.5802/jtnb.591

3. Edwards, H.M.: Riemann's Zeta Function. Dover Publications (2001)

4. Robin, G.: Grandes valeurs de la fonction somme des diviseurs et hypothèse de Riemann. J. Math. pures appl 63(2), 187-213 (1984)

5. Rosser, J.B., Schoenfeld, L.: Approximate Formulas for Some Functions of Prime Numbers. Illinois Journal of Mathematics 6(1), 64-94 (1962). DOI doi:10.1215/ijm/1255631807 